\title{
STUDY OF AFLATOXIN B1-DESTROYING ACTIVITY OF Gliocladium roseum AND Trichoderma viride AND THEIR ANTAGONISM TOWARD TOXIGENIC Aspergillus flavus
}

\section{L.A. SHCHERBAKOVA, O.D. MIKITYUK, T.A. NAZAROVA, V.G. DZHAVAKHIYA}

All-Russian Research Institute of Phytopathology, Federal Agency of Scientific Organizations, 5, ul. Institute, pos. Bol'shie Vyazemy, Odintsovskii Region, Moscow Province, 143050 Russia, e-mail dzhavakhiya@yahoo.com, larisa@vniif.ru

Acknowledgements:

Supported financially by Russian Science Foundation (RSF project № 14-16-00150)

Received February 9, 2016

\section{Abstract}

Aflatoxin B1-destroying activity and antagonistic potential of Gliocladium roseum GRZ7 and Trichoderma viride TV35 strains isolated from natural substrates colonized by aflatoxigenic Aspergillus flavus were studied in vitro. Submerged cultures of G. roseum grown on liquid Czapek's medium with casein hydrolizate (Czapek-CasH) at $28^{\circ} \mathrm{C}$ and $200 \mathrm{rpm}$ for 7 days were able to destroy 80-90\% of aflatoxin B1 (AFB1), which was added in the nutrient medium before inoculation. T. viride grown under the same conditions destroyed only $48 \%$ of initial AFB1 during the same time of cultivation. The tested $T$. viride strain effectively suppressed the growth of toxigenic $A$. flavus strain A11 on Czapek-CasH agar. Co-cultivation of A11 with T. viride TV35 resulted in $64 \%$ diminution of the average colony diameter of the aflatoxigenic strain. The strain GRZ7 of G. roseum was ineffective as an antagonist of A11. AFB1-destroyimg activity was detected in samples of high-molecular weight metabolites ( $>5 \mathrm{kDa}$ ) isolated from culture liquid of $G$. roseum grown without AFB1. In addition, $T$. viride ability to degrade the mycotoxin was shown to be inducible. Obtained results were supposed to be of interest for further investigation on decontamination of feeds, which are contaminated with AFB1 or AFB1-producers.

Keywords: aflatoxin B1, biological decontamination, Gliocladium roseum, Trichoderma viride

Contamination of forage grains and plant materials with aflatoxins, the secondary metabolites of aspergillus fungi, is a serious problem. Because of hepatotoxicity, carcinogenicity and teratogenicity, aflatoxins are dangerous for the vast majority of warm-blooded organisms [1]. Feed contamination with aflatoxins can cause animal death or decreased production, and also lead to food stuff contamination [2]. Due to wide spread of aspergillas, their ability to evolve as facultative parasites on vegetating plants and, at the same time, to keep up saprophytic growth, it is almost impossible to completely exclude the contamination of grain and fodder grass by aflatoxin producers during harvesting and storage. Therefore, the approaches to solving the problem are mainly aimed at decontamination of raw materials [3, 4] or biocontrol of potentially aflatoxigenic Aspergillium species, in particular Aspergillus flavus, by microbial antagonists [5]. Due to the high stability of aflatoxins, their chemical or physical degradation is carried out under stringent conditions $[6,7]$. This often reduces the feed quality making such treatment economically unprofitable. The biological method of decontamination [8-10] is based on the use of secondary metabolites of some plants toxic to A. flavus [11], and also on the search for natural inhibitors of aflatoxinogenesis or microorganism that could serve as a source of enzymes destroying aflatoxins or transform them to non-hazardous derivatives [12-16].

For example, when some micromycetes colonizing natural substrates are grown together with the toxigenic isolates of $A$. flavus, aflatoxin B1 (AFB1), typ- 
ical for this fungus, decomposes in the culture liquid (CL) [17]. The enzymatic nature of the detoxifying activity of CL in a such biodestructor, Phoma glomerata (strain PG41), has been confirmed [18].

Here we first investigated the ability of two other micromycetes, Gliocladium roseum and Trichoderma viride, previously isolated from the toxigenic A. flavus consortium, to destroy AFB1 in view to assess the prospects of their use as sources of the AFB1-catabolizing enzymes or as antagonists of the of AFB1 producer.

Technique. Destroying activity of GRZ7 (Gliocladium roseum Bainier) and TV35 (Trichoderma viride Pers.) strains from the collection of Laboratory of Pathophysiology (All-Russian Research Institute of Phytopathology) was studied in 7-day cultures grown in liquid Czapek's medium with casein hydrolizate (Czapek-CasH) at $28{ }^{\circ} \mathrm{C}$ and $200 \mathrm{rpm}$ (Excella ${ }^{\mathrm{TM}}$ E-25/25R, New Brunswick Scientific Co., Inc., USA). Prior to fungi inoculation, AFB1 $(5-10 \mu \mathrm{g} / \mathrm{ml}$, Sigma», USA) was added under sterile conditions. High molecular weight metabolites with nominally cut off molecular weight of $>5 \mathrm{kDa}$ were isolated from CL of $G$. roseum, grown under the same conditions in the absence of toxin (intact CL), by precipitation of the filtrate with ammonium sulfate and ultrafiltration of aqueous solution of the precipitate. AFB1 was added to sterile samples of the high molecular weight fraction and the mixture was incubated at $27-28{ }^{\circ} \mathrm{C}$ for 3 days. Residual amounts of B1 in CL or in the sample of the fraction were determined using high performance liquid chromatography [18].

The effect of $T$. viride and $G$. roseum on the growth of toxigenic A. flavus (strain A11) was studied by the method of double cultures (co-cultivation on potato-glucose agar at $20-22{ }^{\circ} \mathrm{C}$ for 6 days). The minimum and maximum diameters were measured, and the area of the colonies was calculated based on the maximum diameter. Control cultures of strain A11 were grown under the same conditions in the absence of the putative antagonists.

Data were processed using Statistica 6.0 (StatSoft, USA). The significance of the differences between control and experimental values was confirmed by a $t$-test for independent variables $(\mathrm{p} \leq 0.05)$. The table and the figure show the mean $(M)$ and standard error of the mean $(m)$. Each experiment included at least 6 replicates with 3-fold reproduction.

Results. A study of the dynamics of destruction of aflatoxin by G. roseum showed that after 3 days of its growth on medium with AFB1 the content of the latter in CL was halved, and by the end of cultivation it did not exceed 10-20\% of the initial amount (Fig., curve 4). A significant decrease in the AFB1 concentration also occurred after its incubation in the fraction of high-molecular metabolites isolated from the filtrate of intact CL of G. roseum (see Fig., curve 5). Thermal treatment of the fraction led to the loss of toxin-degrading activity (see Fig., curve 1).

These results suggest that extracellular enzymes of this biodestructor may participate in the decomposition of AFB1, similar to that in P. glomerata [18] or other ascomycetes $[12,19]$. Earlier, it was found that $G$. roseum produces zearalenone-specific lactonase [20] which hydrolyses the lactone ring in ZEN, the mycotoxin of fusarium fungi dangerous for mammalians, thereby reducing ZEN toxicity [21]. The inhibition of most micromycetes by ZEN significantly reduces the possibility of using antagonistic fungi against toxic fusariums, which affect plants, but G. roseum is not sensitive to ZEN [20]. Like ZEN, aflatoxin B1 molecule contains the lactone ring which is associated with its toxicity, mutagenic and carcinogenic effects [22-25]. Aflatoxin-destroying activity that we have discovered in $G$. roseum, makes it even more attractive as a bioagent against fungi 
that produce mycotoxins of different chemical nature.

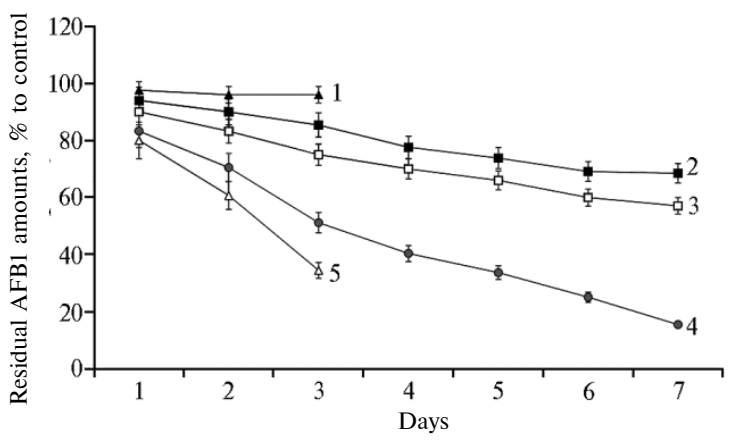

Aflatoxin B1 (AFB1) biodegradation by the micromycetes Gliocladium roseum GRZ7 (4) and Trichoderma viride TV35 (2, 3) grown on the medium with aflatoxin, and intact (5) or heat inactivated (1) high molecular extracellular metabolites of $G$. roseum. Controls: for 2, 3, 4- the AFB1 concentration in a nutrient medium not inoculated with micromycetes; for $1,5-$ the AFB1 amount immediately after its adding to analyzed fraction of metabolites (without incubation). Differences between the values in treated and control samples are statistically significant at $\mathrm{P} \leq 0.05$; the

Y-errors bars show SEM for 3 experiments with 6 replicates per option in each.

The destroying activity of the studied $T$. viride strain was low. The utilization of AFB1 from the nutrient medium occurred slowly, and its concentration in the CL decreased not significantly as the fungus grew (see Fig., curve 2). However, the ability of the strain to destroy AFB1 in LC increased (curve 3) in case of using spore suspension of the fungus previously grown on the CzapekCasH agar medium supplemented with AFB1 $(0.9 \mu \mathrm{g} / \mathrm{ml})$. When using such an inoculum, the proportion of the destructured toxin in the immersed culture increased by the end of the fermentation by $20 \%(\mathrm{p}=0.03)$. Therefore, the AFB1-degrading activity which we detected in $T$. viride was inducible and could be increased by mutagenesis and selection of the most effective clones. Moreover, the tested $T$. viride strain proves to be an effective antagonist of A. flavus. When the toxigenic A11 strain was grown together with $T$. viride, the growth of the aflatoxin producer was significantly lower (Table) with a reduction in the colony size by $64 \%$ on average.

Radial growth of toxigenic Aspergillus flavus A11 colonies in single culture and under co-cultivation with Trichoderma viride TV35 (Tv) or Gliocladium roseum GRZ7 $(G r)(M \pm m)$

\begin{tabular}{l|c|c|c|c|c}
\hline \multirow{2}{*}{$\begin{array}{l}\text { Variant of } \\
\text { cultivation }\end{array}$} & \multicolumn{2}{|c|}{ Average colony diameter, $\mathrm{cm}$} & \multirow{2}{*}{$\begin{array}{c}\text { Average colony } \\
\text { area, } \mathrm{cm}^{2}\end{array}$} & $\begin{array}{c}\text { Growth inhibition, } \\
\text { \% to control value }\end{array}$ & $\mathrm{p}$ \\
\cline { 2 - 3 } A11 (control) & $\min$ & $\max$ & $39.50 \pm 0.00$ & & \\
A11 $+T v$ & $6.90 \pm 0.03$ & $3.10 \pm 0.00$ & $14.25 \pm 0.56$ & 63.9 & 0.004 \\
A11 $+G r$ & $6.20 \pm 0.06$ & $6.89 \pm 0.06$ & $37.27 \pm 0.12$ & 5.6 & 0.05 \\
\hline
\end{tabular}

Mycoparasite $G$. roseum did not noticeabley influence the radial growth of A. flavus colonies as compared to the control, however, the differences were significant (Table), and in some cases the A11 strain colonies were overgrow with the $G$. roseum mycelium by the end of co-cultivation which could be a sign of hyperparasitism.

Thus, it has been found out that the studied strains of Tricho-derma viride and Gliocladium roseum, the antagonist species of many pathogenic fungi, competing with Aspergillus flavus for natural substrates, were different in their capacity to degrade the aflatoxin B1 in vitro and to inhibit the growth of aflatoxigenic A. flavus strain. Additionally, the inducibility of degrading activity, discovered by us, is of undoubted interest both in the theoretical and practical aspects. The obtained results can be used in the development of technologies for biological decontamination of feed contaminated with both aflatoxin B1 and its producer. In this case, $G$. roseum, apparently, can serve as a source of enzymes that decompose this mycotoxin, and $T$. viride can be a bioagent that inhibits the development of a producer. 


\section{R E F E R E N C E S}

1. Bbosa G.S., Kitya D., Lubega A., Ogwal-Okeng J., Anokbonggo W.W., $\mathrm{K}$ y e g o m b e D.B. Review of the biological and health effects of aflatoxins on body organs and body systems. In: Aflatoxins: recent advances and future prospects. M. Razzaghi-Abyaneh (ed.). InTech, Rijeka, Croatia, 2013: 239-266 (doi: 10.5772/51201).

2. Fil a s i A., S i r e 1 i U.T. Occurrence of aflatoxins in food. In: Aflatoxins: recent advances and future prospects. M. Razzaghi-Abyaneh (ed.). InTech, Rijeka, Croatia, 2013: 143-170 (doi: 10.5772/51031).

3. Mille r J.D., Schaafsma A.W., Bhatnagar D., Bondy G., Carbone I., Har ris L.J., Harrison G., Munkvold G.P., Oswald I.P., P e s t k a J.J., Sharpe L., $\mathrm{Sum}$ a rah M.W., Tit t le mi e r S.A., $\mathrm{Z}$ hou T. Mycotoxins that affect the North American agrifood sector: state of the art and directions for the future. World Mycotoxin Journal, 2014, 7(1): 63-82.

4. Wh it low L.W. Evaluation of mycotoxin binders. Proc. $4^{\text {th }}$ Mid-Atlantic Nutrition Conference. N.G. Zimmermann (ed.). College Park, University of Maryland, 2006: 132-143.

5. Dhramaputra O.S., Putri A.S.R., Retnovati I., A mbarwati S. Antagonistic effect of three fungal isolates to aflatoxin-producing Aspergillus flavus. Biothropia, 2003, 21: 19-31.

6. R a t e r s M., M a t is s e k R. Thermal stability of aflatoxin B1 and ochratoxin A. Mycotoxin Research, 2008, 24(3): 130-134 (doi: 10.1007/BF03032339).

7. Wo m a ck E.D., B row n ab A.E., S parks a D.L. A recent review of non-biological remediation of aflatoxin-contaminated crops. J. Sci. Food Agric., 2014, 94(9): 1706-1714 (doi: 10.1002/jsfa.6520).

8. Wu Q., Jezkova A., Yuan Z., Pavlikova L., Do hnal V., Kuca K. Biological degradation of aflatoxins. Drug Metab. Rev., 2009, 41(1): 1-7 (doi: 10.1080/03602530802563850).

9. Kabak B., Dobs o n A.D.W., Var I. Strategies to prevent mycotoxin contamination of food and animal feed: A review. Critical Reviews in Food Science and Nutrition, 2006, 46: 593619 (doi: 10.1080/10408390500436185).

10. M is h r a H.N., D a s C. A review on biological control and metabolism of aflatoxin. Critical Reviews in Food Science and Nutrition, 2003, 43: 245-264 (doi: 10.1080/10408690390826518).

11. Shukla R., Kumar A., Prasad C.S., Srivastava B., Dubey N.K. Antimycotic and antiaflatoxigenic potency of Adenocalymma alliaceum Miers. on fungi causing biodeterioration of food commodities and raw herbal drugs. Int. Biodeter. Biodegr., 2008, 62: 348-351 (doi: 10.1016/j.ibiod.2007.11.006).

12. Sardjono E., Rahay u S., Raharjo S., Rahay u K. Detoxification of aflatoxin B1 by extracellular enzymes of Aspergillus oryzae KKB4. Indonesian Food and Nutrition Progress, 2004, 11(1): $30-34$.

13. Motomura M., Toyomasu T., Mizuno 1 K., Shinozawa 1 T. Purification and characterization of an aflatoxin degradation enzyme from Pleurotus ostreatus. Microbiol. Res., 2003, 158: 237-242 (doi: 10.1078/0944-5013-00199).

14. Albe rts J.F. Microbial degradation of mycotoxins. PhD Thesis. University of Stellenbosch, 2007.

15. Guan S., Ji C., Zhou T., Li J.X., M a Q.G, Niu T.G. Aflatoxin B1 degradation by Stenotrophomonas maltophilia and other microbes selected using coumarin medium. Int. J. Mol. Sci., 2008, 9: 1489-1503 (doi: 10.3390/ijms9081489).

16. Ca o H., Li u D., Mo X., Xi e C., Y a o D. A fungal enzyme with the ability of aflatoxin B1 conversion: purification and ESI-MS/MS identification. Microbiol. Res., 2011, 166: 475-483 (doi: 10.1016/j.micres.2010.09.002).

17. Zhemchuzhina N., Mikityuk O., Shcherbakova L., Campbell B., Dorofe eva L., D z havakhiy a V. Isolation of aflatoxin B1-degrading fungi among the mycoflora coincidental with toxigenic Aspergillus flavus. Proc. 14 ${ }^{\text {th }}$ Int. Congr. on Molecular PlantMicrobe Interactions. Quebec City, 2009: 158.

18. Shcherbakova L., Statsyuk N., Mikityuk O., Nazarova T., Dzhavakhiy a V. Aflatoxin B1-degrading activity by Phoma glomerata PG41 isolated from natural substrate colonized by aflatoxigenic Aspergillus flavus. Jundishapur Journal of Microbiology, 2015, 8(1): 1-5 (doi: 10.5812/jjm.24324).

19. Forootanfara H., Faramarzia M.A., Shahverdia A.R., Yazdia M.T. Purification and biochemical characterization of extracellular laccase from the ascomycete Paraconiothyrium variabile. Bioresource Technol., 2011, 10(2): 1808-1814 (doi: 10.1016/j.biortech.2010.09.043).

20. Ute r mark J., Karlovsky P. Role of zearalenone lactonase in protection of Gliocladium roseum from fungitoxic effects of the mycotoxin zearalenone. Appl. Environ. Microb., 2007, 73(2): 637-642 (doi: 10.1128/AEM.01440-06).

21. Utermark J., Karlovsky P. Detoxification of zearalenone by Gliocladium roseum: biological function and regulation of zearalenone lactonase synthesis. Proc. 29th Mycotoxin Workshop. Fellbach, 2007: 54.

22. Lee L.S., Dunn J.J., D e Luc c a A.J., C i e g le r A. Role of lactone ring of aflatoxin B1 
in toxicity and mutagenicity. Experientia, 1981, 37: 16-17 (doi: 10.1007/BF01965543).

23. Gu a n S., Z h o u T., Y m Y., X i e M., R u a n Z., Y o u n g J.C. Microbial strategies to control aflatoxins in food and feed. World Mycotoxin Journal, 2011, 4: 413-424 (doi: 10.3920/WMJ2011.1290

24. Nicolás-Vázquez I., Méndez-Albores A., Moreno-Martínez E., Mi ra nd a R., Castro M. Role of lactone ring in structural, electronic, and reactivity properties of aflatoxin B1: a theoretical study. Arch. Environ. Con. Tox., 2010, 59(3): 393-406 (doi: 10.1007/s00244-010-9501-x).

25. I r a m W., Anjum T., I q b a l M., G haffar A., Abbas M. Mass spectrometric identification and toxicity assessment of degraded products of aflatoxin B1 and B2 by Corymbia citriodora aqueous extracts. Scientific Reports, 2015, 5: 14672 (doi: 10.1038/srep14672). 